\title{
THE AMERICAN STRADDLE CLOSE TO EXPIRY
}

\author{
GHADA ALOBAIDI AND ROLAND MALLIER
}

Received 23 August 2005; Revised 26 December 2005; Accepted 22 March 2006

We address the pricing of American straddle options. We use a technique due to Kim (1990) to derive an expression involving integrals for the price of such an option close to expiry. We then evaluate this expression on the dual optimal exercise boundaries to obtain a set of integral equations for the location of these exercise boundaries, and solve these equations close to expiry.

Copyright (c) 2006 G. Alobaidi and R. Mallier. This is an open access article distributed under the Creative Commons Attribution License, which permits unrestricted use, distribution, and reproduction in any medium, provided the original work is properly cited.

\section{Introduction}

One of the classic problems of mathematical finance is the pricing of American options and the behavior of the optimal exercise boundary close to expiry. For the uninitiated, financial derivatives are securities whose value is based on the value of some other underlying security, and options are an example of derivatives, carrying the right but not the obligation to enter into a specified transaction in the underlying security. A call option allows the holder to buy the underlying security at a specified strike price $E$, a put option allows the holder to sell the underlying at the price $E$, while a straddle, which we consider in the current study, allows the holder the choice of either buying or selling (but not both) the security. If $S$ is the price of the underlying, then the payoff at expiry is $\max (S-E, 0)$ for a call, $\max (E-S, 0)$ for a put, and $\max (S-E, E-S)$ for a straddle. From this payoff, it would appear at first glance that the holder of a straddle is holding a call and a put on the same underlying with the same strike and the same expiry, but is only allowed to exercise one. This is true for a European straddle, which can be exercised at expiry, because if the call is in the money, the put must be out of the money and vice versa, and the holder will naturally exercise whichever of the call and the put is in the money at expiry, unless he is unlucky enough that $S=E$ so that they are both exactly at the money, and the payoff is zero. 
However, an American straddle is not simply the combination of an American call and put. Unlike Europeans, which can be exercised only at expiry, American options may be exercised at any time at or before expiry. The payoff from immediate exercise is the same as the payoff at expiry, namely, $\max (S-E, 0)$ for a call, $\max (E-S, 0)$ for a put, and $\max (S-E, E-S)$ for a straddle. Naturally, a rational investor will choose to exercise early if that maximizes his return, and it follows that there will be regions where it is optimal to hold the option, and others where exercise is optimal, with a free boundary known as the optimal exercise boundary separating these regions. It is because the free boundaries for the straddle differ from those for the call and the put so that an American straddle differs from the combination of an American call and put.

For vanilla Americans, there have been numerous studies of this free boundary, but a closed form solution for its location remains elusive, as does a closed form expression for the value of an American option. One popular approach [11, 16, 20] has been used to reformulate the problem as an integral equation for the location of the free boundary, which can be solved using either asymptotics or numerics, although for problems with a single free boundary, such as vanilla American calls and puts, it may be simpler just to apply asymptotics directly to the Black-Scholes-Merton partial differential equation using the methods developed by Tao [23] for Stefan problems, and this has been done for the American call and put $[1,8,18]$. For the American straddle, which we have previously studied using partial Laplace transforms [2], there are two free boundaries not one: an upper one on which an exercise as a call occurs, and a lower one on which an exercise as a put occurs, and applying Tao's method is somewhat harder, making the integral equation approach more attractive.

We should also mention the work of Kholodnyi [14, 15] on American-style options with general payoffs of which the American straddle considered here is one particular example. In [14], a new formulation in terms of the semilinear evolution equation in the entire domain of the state variables was introduced, while in [15], the foreign exchange option symmetry was introduced.

In the present study, we will use an approach originally developed for physical Stefan problems [17] and later applied to economics [20], and applied to vanilla Americans with great success by Kim [16] and Jacka [11], who independently derived the same results: Kim both by using McKean's formula and by taking the continuous limit of the GeskeJohnson formula [9] which is a discrete approximation for American options, and thereby demonstrating that those two approaches led to the same result, and Jacka by applying probability theory to the optimal stopping problem. P. Carr et al. [6] later used these results to show how to decompose the value of an American option into intrinsic value and time value. The approach in $[11,16,20]$ leads to an integral equation for the location of the free boundary, which was solved numerically by [10] and by approximating the free boundary as a multipiece exponential function by [13].

Our contribution is to extend the analysis of $[11,16,20]$ to the American straddle and obtain a set of integral equations for the locations of the free boundaries. These equations are then solved asymptotically to find the locations of the free boundaries close to expiry, and the results are compared to the results for American calls and puts. Before we proceed with our analysis, we note that [24] proved that the free boundary was regular for vanilla Americans, and his analysis can be carried over to the straddle. 


\section{Analysis}

In this section, we follow the approach taken in [16]. Our starting point is the BlackScholes-Merton partial differential equation $[5,21]$ governing the price $V$ of an equity derivative,

$$
\mathscr{L} V=\left[\frac{\partial}{\partial \tau}-\frac{\sigma^{2} S^{2}}{2} \frac{\partial^{2}}{\partial S^{2}}-(r-D) S \frac{\partial}{\partial S}+r\right] V=0
$$

where $S$ is the price of the underlying stock and $\tau=T-t$ is the time remaining until expiry. In our analysis, the volatility $\sigma$, risk-free interest rate $r$, and dividend yield $D$ are assumed constant. For European options, the value of the option can be written as

$$
V^{E}(S, \tau)=\int_{0}^{\infty} V^{E}(Z, 0) G(S, Z, \tau) d Z
$$

where $V^{E}(S, 0)$ is the payoff at expiry, and we have introduced Green's function,

$$
G(S, Z, \tau)=\frac{e^{-r \tau}}{Z \sigma \sqrt{2 \pi \tau}} \exp \left(-\frac{\left[\ln (S / Z)+r^{(-)} \tau\right]^{2}}{2 \sigma^{2} \tau}\right)
$$

with $r^{(-)}=r-D-\sigma^{2} / 2$ and $r^{(+)}=r-D+\sigma^{2} / 2$. Using (2.2), the price of a European straddle with strike $E$ and payoff at expiry $V^{E}(S, 0)=\max (S-E, E-S)$ is

$$
V^{E}(S, \tau)=\mathrm{Se}^{-D \tau} \operatorname{erf}\left(\frac{\ln (S / E)+r^{(+)} \tau}{\sigma \sqrt{2 \tau}}\right)-E e^{-r \tau} \operatorname{erf}\left(\frac{\ln (S / E)+r^{(-)} \tau}{\sigma \sqrt{2 \tau}}\right)
$$

The value of a European straddle is simply the sum of the values of a European call and put. In the above, erf is the error function, with erfc the complementary error function.

To derive our analytic expression for an American straddle, two paths may be taken. We may follow [16] and approximate an American option by a Bermudan option with exercise opportunities at $m \Delta \tau$ for $0 \leq m \leq n$, and then take the limit $\Delta \tau \rightarrow 0$, so that we can neglect certain terms, with $n \Delta \tau \rightarrow \tau$ to recover the value of the American option. In this procedure, we write the value of the Bermudan option at time $m \Delta \tau$ in terms of its value at $(m-1) \Delta \tau$, where the option will be exercised if its value falls below that from immediate exercise. For the Bermudan option, the upper and lower optimal exercise boundaries at time $m \Delta \tau, S=S_{u}(m \Delta \tau)$ and $S=S_{l}(m \Delta \tau)$ will be the solutions of $V^{B}(S, m \Delta \tau)=S-E$ and $V^{B}(S, m \Delta \tau)=E-S$, respectively, and we will hold the option if $S_{l}(m \Delta \tau)<S<S_{u}(m \Delta \tau)$ but exercise as a call if $S>S_{u}(m \Delta \tau)$ and as a put if $S<S_{l}(m \Delta \tau)$. To leading order in $\Delta \tau$, we find

$$
\begin{aligned}
V^{B}(S, n \Delta \tau)= & V^{E}(S, n \Delta \tau)+\sum_{m=1}^{n-1} \Delta \tau \int_{S_{f}(m \Delta \tau)}^{\infty}(D Z-r E) G(S, Z,(n-m) \Delta \tau) d Z \\
& +\sum_{m=1}^{n-1} \Delta \tau \int_{0}^{S_{l}(m \Delta \tau)}(r E-D Z) G(S, Z,(n-m) \Delta \tau) d Z .
\end{aligned}
$$


To go to the continuous exercise American case, we follow [16] and take the limit $\Delta \tau \rightarrow 0$ with $n \Delta \tau \rightarrow \tau$, using $S=S_{u}(\tau)$ and $S_{l}(\tau)$ to denote upper and lower optimal exercise boundaries, respectively,

$$
\begin{aligned}
V^{A}(S, \tau)= & V^{E}(S, \tau)+\int_{0}^{\tau} \int_{S_{u}(\zeta)}^{\infty}(D Z-r E) G(S, Z, \tau-\zeta) d Z d \zeta \\
& +\int_{0}^{\tau} \int_{0}^{S_{l}(\zeta)}(r E-D Z) G(S, Z, \tau-\zeta) d Z d \zeta
\end{aligned}
$$

An alternative approach is to apply a more general formula. For American-style options with early exercise features, it follows from the work of $[4,11,12,16,17,20]$ that if such an option obeys (2.1), where it is optimal to hold the option and the payoff at expiry is $V(S, 0)$ while that from immediate exercise is $P(S, \tau)$, then we can write the value of the option as the sum of the value of the corresponding European option $V^{(e)}(S, \tau)$ together with another term representing both the premium from an early exercise, a technique introduced for the American call and put by $[4,12]$,

$$
V(S, \tau)=V^{(e)}(S, \tau)+\int_{0}^{\tau} \int_{0}^{\infty} \mathscr{F}(Z, \zeta) G(S, Z, \tau-\zeta) d Z d \zeta,
$$

with $\mathscr{F}(S, \tau) \equiv 0$ where it is optimal to hold the option while where exercise is optimal $\mathscr{F}(S, \tau)$ is the result of substituting the early exercise payoff $P(S, \tau)$ into $(2.1), \mathscr{F}(S, \tau)=$ $\mathscr{L} P$. For the straddle, $\mathscr{F}=D S-r E$ when we exercise as a call, and $\mathscr{F}=r E-D S$ when we exercise as a put.

Using either of these approaches, we find that

$$
\begin{array}{r}
V^{A}(S, \tau)=\operatorname{Se}^{-D \tau} \operatorname{erf}\left(\frac{\ln (S / E)+r^{(+)} \tau}{\sigma \sqrt{2 \tau}}\right)-E e^{-r \tau} \operatorname{erf}\left(\frac{\ln (S / E)+r^{(-)} \tau}{\sigma \sqrt{2 \tau}}\right) \\
+\frac{1}{2} \int_{0}^{\tau}\left[\operatorname { S D e } { } ^ { - D ( \tau - \zeta ) } \left(\operatorname{erf}\left[\frac{\ln \left(S / S_{u}(\zeta)\right)+r^{(+)}(\tau-\zeta)}{\sigma \sqrt{2(\tau-\zeta)}}\right]\right.\right. \\
\left.+\operatorname{erf}\left[\frac{\ln \left(S / S_{l}(\zeta)\right)+r^{(+)}(\tau-\zeta)}{\sigma \sqrt{2(\tau-\zeta)}}\right]\right) \\
-r E e^{-r(\tau-\zeta)}\left(\operatorname{erf}\left[\frac{\ln \left(S / S_{u}(\zeta)\right)+r^{(-)}(\tau-\zeta)}{\sigma \sqrt{2(\tau-\zeta)}}\right]\right. \\
\left.\left.+\operatorname{erf}\left[\frac{\ln \left(S / S_{l}(\zeta)\right)+r^{(-)}(\tau-\zeta)}{\sigma \sqrt{2(\tau-\zeta)}}\right]\right)\right] d \zeta,
\end{array}
$$

which is an expression for the value of the American straddle. If we compare our results to the expressions for the American call and put in $[11,16]$, the expressions involving $S_{u}(\tau)$ appear in the expression for the call while those with $S_{l}(\tau)$ appear in that for the put, and at first glance, it looks as though the value of an American straddle is the sum of the values of an American call and an American put, as was the case with the Europeans, although of course this is not really the case as the free boundaries for the straddle will differ from those for the call and the put. 


\section{Integral equations}

The integral equations for the location of the upper and lower free boundaries $S=S_{u}(\tau)$ and $S=S_{l}(\tau)$ are derived by substituting the expression for the American straddle $(2.8)$ into the conditions at the free boundaries, and requiring that the value must be continuous across the boundaries, so that $V^{A}=S-E$ at $S=S_{u}(\tau)$ and $V^{A}=E-S$ at $S=S_{l}(\tau)$, together with the high contact or smooth pasting condition [22] that $\left(\partial V^{A} / \partial S\right)=1$ at $S_{u}(\tau)$ and $\left(\partial V^{A} / \partial S\right)=-1$ at $S_{l}(\tau)$. These four conditions will give us four integral equations, which as discussed in $[16,17]$ are Volterra equations of the second kind.

For the upper boundary, the condition that $V^{A}=S-E$ at $S=S_{u}(\tau)$ yields

$$
\begin{gathered}
S_{u}(\tau)\left[1-e^{-D \tau} \operatorname{erf}\left(\frac{\ln \left(S_{u}(\tau) / E\right)+r^{(+)} \tau}{\sigma \sqrt{2 \tau}}\right)\right]-E\left[1-e^{-r \tau} \operatorname{erf}\left(\frac{\ln \left(S_{u}(\tau) / E\right)+r^{(-)} \tau}{\sigma \sqrt{2 \tau}}\right)\right] \\
=\int_{0}^{\tau}\left(\frac { S _ { u } ( \tau ) D e ^ { - D ( \tau - \zeta ) } } { 2 } \left[\operatorname{erf}\left(\frac{\ln \left(S_{u}(\tau) / S_{u}(\zeta)\right)+r^{(+)}(\tau-\zeta)}{\sigma \sqrt{2(\tau-\zeta)}}\right)\right.\right. \\
\left.\quad+\operatorname{erf}\left(\frac{\ln \left(S_{u}(\tau) / S_{l}(\zeta)\right)+r^{(+)}(\tau-\zeta)}{\sigma \sqrt{2(\tau-\zeta)}}\right)\right] \\
-\frac{r E e^{-r(\tau-\zeta)}}{2}\left[\operatorname{erf}\left(\frac{\ln \left(S_{u}(\tau) / S_{u}(\zeta)\right)+r^{(-)}(\tau-\zeta)}{\sigma \sqrt{2(\tau-\zeta)}}\right)\right. \\
\left.\left.+\operatorname{erf}\left(\frac{\ln \left(S_{u}(\tau) / S_{l}(\zeta)\right)+r^{(-)}(\tau-\zeta)}{\sigma \sqrt{2(\tau-\zeta)}}\right)\right]\right) d \zeta,
\end{gathered}
$$

while for the lower boundary, the condition that $V^{A}=E-S$ at $S=S_{l}(\tau)$ yields

$$
\begin{gathered}
-S_{l}(\tau)\left[1+e^{-D \tau} \operatorname{erf}\left(\frac{\ln \left(S_{l}(\tau) / E\right)+r^{(+)} \tau}{\sigma \sqrt{2 \tau}}\right)\right]+E\left[1+e^{-r \tau} \operatorname{erf}\left(\frac{\ln \left(S_{l}(\tau) / E\right)+r^{(-)} \tau}{\sigma \sqrt{2 \tau}}\right)\right] \\
=\int_{0}^{\tau}\left(\frac { S _ { l } ( \tau ) D e ^ { - D ( \tau - \zeta ) } } { 2 } \left[\operatorname{erf}\left(\frac{\ln \left(S_{l}(\tau) / S_{u}(\zeta)\right)+r^{(+)}(\tau-\zeta)}{\sigma \sqrt{2(\tau-\zeta)}}\right)\right.\right. \\
\left.\quad+\operatorname{erf}\left(\frac{\ln \left(S_{l}(\tau) / S_{l}(\zeta)\right)+r^{(+)}(\tau-\zeta)}{\sigma \sqrt{2(\tau-\zeta)}}\right)\right] \\
-\frac{r E e^{-r(\tau-\zeta)}}{2}\left[\operatorname{erf}\left(\frac{\ln \left(S_{l}(\tau) / S_{u}(\zeta)\right)+r^{(-)}(\tau-\zeta)}{\sigma \sqrt{2(\tau-\zeta)}}\right)\right. \\
\left.\left.+\operatorname{erf}\left(\frac{\ln \left(S_{l}(\tau) / S_{l}(\zeta)\right)+r^{(-)}(\tau-\zeta)}{\sigma \sqrt{2(\tau-\zeta)}}\right)\right]\right) d \zeta
\end{gathered}
$$


6 The American straddle close to expiry

while the condition $\left(\partial V^{A} / \partial S\right)=1$ at $S=S_{u}(\tau)$ gives

$$
\begin{aligned}
1-e^{-D \tau}\left[\operatorname{erf}\left(\frac{\ln \left(S_{u}(\tau) / E\right)+r^{(+)} \tau}{\sigma \sqrt{2 \tau}}\right)-\frac{\sqrt{2}}{\sigma \sqrt{\pi \tau}} \exp \left(-\frac{\left[\ln \left(S_{u}(\tau) / E\right)+r^{(+)} \tau\right]^{2}}{2 \sigma^{2} \tau}\right)\right] \\
+\frac{E \sqrt{2} e^{-r \tau}}{S_{u}(\tau) \sigma \sqrt{\pi \tau}} \exp \left(-\frac{\left[\left(S_{u}(\tau) / E\right)+r^{(-)} \tau\right]^{2}}{2 \sigma^{2} \tau}\right) \\
=\int_{0}^{\tau}\left(\frac { D e ^ { - D ( \tau - \zeta ) } } { 2 } \left[\operatorname{erf}\left(\frac{\ln \left(S_{u}(\tau) / S_{u}(\zeta)\right)+r^{(+)}(\tau-\zeta)}{\sigma \sqrt{2(\tau-\zeta)}}\right)\right.\right. \\
\left.\quad+\operatorname{erf}\left(\frac{\ln \left(S_{u}(\tau) / S_{l}(\zeta)\right)+r^{(+)}(\tau-\zeta)}{\sigma \sqrt{2(\tau-\zeta)}}\right)\right]+\frac{D e^{-D(\tau-\zeta)}}{\sigma \sqrt{2 \pi(\tau-\zeta)}} \\
\times \quad\left[\exp \left(-\frac{\left[\ln \left(S_{u}(\tau) / S_{u}(\zeta)\right)+r^{(+)}(\tau-\zeta)\right]^{2}}{2 \sigma^{2}(\tau-\zeta)}\right)\right. \\
\left.\quad+\exp \left(-\frac{\left[\ln \left(S_{u}(\tau) / S_{l}(\zeta)\right)+r^{(+)}(\tau-\zeta)\right]^{2}}{2 \sigma^{2}(\tau-\zeta)}\right)\right]-\frac{r E e^{-r(\tau-\zeta)}}{S_{u}(\tau) \sigma \sqrt{2 \pi(\tau-\zeta)}} \\
\times \\
\quad\left[\exp \left(-\frac{\left[\ln \left(S_{u}(\tau) / S_{u}(\zeta)\right)+r^{(-)}(\tau-\zeta)\right]^{2}}{2 \sigma^{2}(\tau-\zeta)}\right)\right. \\
\left.\quad+\exp \left(-\frac{\left[\ln \left(S_{u}(\tau) / S_{l}(\zeta)\right)+r^{(-)}(\tau-\zeta)\right]^{2}}{2 \sigma^{2}(\tau-\zeta)}\right)\right] d \zeta
\end{aligned}
$$

and the condition $\left(\partial V^{A} / \partial S\right)=-1$ at $S_{l}(\tau)$ gives

$$
\begin{aligned}
-1-e^{-D \tau}\left[\operatorname{erf}\left(\frac{\ln \left(S_{l}(\tau) / E\right)+r^{(+)} \tau}{\sigma \sqrt{2 \tau}}\right)-\frac{\sqrt{2}}{\sigma \sqrt{\pi \tau}} \exp \left(-\frac{\left[\ln \left(S_{l}(\tau) / E\right)+r^{(+)} \tau\right]^{2}}{2 \sigma^{2} \tau}\right)\right] \\
+\frac{E \sqrt{2} e^{-r \tau}}{S_{l}(\tau) \sigma \sqrt{\pi \tau}} \exp \left(-\frac{\left[\ln \left(S_{l}(\tau) / E\right)+r^{(-)} \tau\right]^{2}}{2 \sigma^{2} \tau}\right) \\
=\int_{0}^{\tau}\left(\frac { D e ^ { - D ( \tau - \zeta ) } } { 2 } \left[\operatorname{erf}\left(\frac{\ln \left(S_{l}(\tau) / S_{u}(\zeta)\right)+r^{(+)}(\tau-\zeta)}{\sigma \sqrt{2(\tau-\zeta)}}\right)\right.\right. \\
\left.+\operatorname{erf}\left(\frac{\ln \left(S_{l}(\tau) / S_{l}(\zeta)\right)+r^{(+)}(\tau-\zeta)}{\sigma \sqrt{2(\tau-\zeta)}}\right)\right]+\frac{D e^{-D(\tau-\zeta)}}{\sigma \sqrt{2 \pi(\tau-\zeta)}}
\end{aligned}
$$




$$
\begin{aligned}
& \times\left[\exp \left(-\frac{\left[\ln \left(S_{l}(\tau) / S_{u}(\zeta)\right)+r^{(+)}(\tau-\zeta)\right]^{2}}{2 \sigma^{2}(\tau-\zeta)}\right)\right. \\
& \left.\quad+\exp \left(-\frac{\left[\ln \left(S_{l}(\tau) / S_{l}(\zeta)\right)+r^{(+)}(\tau-\zeta)\right]^{2}}{2 \sigma^{2}(\tau-\zeta)}\right)\right]-\frac{r E e^{-r(\tau-\zeta)}}{S_{l}(\tau) \sigma \sqrt{2 \pi(\tau-\zeta)}} \\
& \times\left[\exp \left(-\frac{\left[\ln \left(S_{l}(\tau) / S_{u}(\zeta)\right)+r^{(-)}(\tau-\zeta)\right]^{2}}{2 \sigma^{2}(\tau-\zeta)}\right)\right. \\
& \left.\quad+\exp \left(-\frac{\left[\ln \left(S_{l}(\tau) / S_{l}(\zeta)\right)+r^{(-)}(\tau-\zeta)\right]^{2}}{2 \sigma^{2}(\tau-\zeta)}\right)\right] d \zeta
\end{aligned}
$$

The expressions (3.1)-(3.4), valid for $\tau \geq 0$, are the integral equations for the location of the free boundaries for the straddle, and each involves the locations of both free boundaries, so that the boundaries are coupled together. If we compare our results to the corresponding expressions for the American call and put, we see that (3.1) appears at first glance to be the sum of the corresponding equations for the call and the put evaluated at the upper boundary while (3.2) appears to be the same expression evaluated at the lower boundary, with a similar relation between (3.3) and (3.4) and the equations coming from the deltas of the call and the put. Once again, we would stress that since the free boundaries for the straddle differ from those for the call and the put, the relation between these equations is not quite so straightforward.

\section{Solution of the integral equations close to expiry}

We will solve the above integral equations (3.1)-(3.4) close to expiry to find expressions for the location of the free boundaries in the limit $\tau \rightarrow 0$, writing $S_{u}(\tau)=S_{u 0} e^{x_{u}(\tau)}$ and $S_{l}(\tau)=S_{l 0} e^{x_{l}(\tau)}$, where $S_{u 0}$ and $S_{l 0}$ are the locations of the upper and lower free boundaries at expiry, which can be deduced by considering the behavior of $\left(\partial V^{A} / \partial \tau\right)$ at expiry. Initially, we will try a solution of the form

$$
\begin{aligned}
& x_{u}(\tau) \sim \sum_{n=1}^{\infty} x_{u n} \tau^{n / 2}, \\
& x_{l}(\tau) \sim \sum_{n=1}^{\infty} x_{l n} \tau^{n / 2},
\end{aligned}
$$

which is motivated both by earlier work on American options and by the work of [23] on Stefan problems in general. Since there will be several terms of the form $\ln \left(S_{u 0} / E\right)$ and $\ln \left(S_{l 0} / E\right)$, we would expect the behavior when $S_{u 0}=E$ to differ from that when $S_{u 0} \neq E$, and similarly with $S_{l 0}$, and this suggests that we consider several cases separately.

Case $4.1(D<r)$. The free boundary starts at $S_{l 0}=E$ and $S_{u 0}=r E / D$. If we take the limit $\tau \rightarrow 0$, we can drop certain terms and (3.1)-(3.4) decouple, so that we have two pairs of equations: one involving only $x_{u}$ but not $x_{l}$, and a second pair involving only $x_{l}$ but not $x_{u}$. Because close to expiry, the value of the put-like element of the straddle is not felt at $S_{u 0}=r E / D$, the pair of equations involving only $x_{u}$ is identical to the pair of integral 
equations for an American call with $D<r$. Recalling that we can decompose an American option into European component and an early exercise component, the pair of equations involving only $x_{l}$ is similar to but not quite identical to the pair of integral equations for an American put with $D<r$ in the limit $\tau \rightarrow 0$. Because close to expiry, the value of the early exercise component of the call-like element of the straddle (2.8) is not felt at $S_{l 0}=E$ but the European component most definitely is.

This decoupling only happens when we take the limit $\tau \rightarrow 0$ and for larger times, the two boundaries remain coupled together. The effect of this decoupling, however, is that for the case $D<r$, in the limit $\tau \rightarrow 0$, the upper free boundary $x_{u}$, which starts above the strike price $E$ at $r E / D$, behaves exactly like the free boundary for the call while the lower free boundary $x_{l}$, which starts at the strike price $E$, behaves at leading order like the free boundary for the put. We would stress that this only holds true in the $\tau \rightarrow 0$ when the boundaries are decoupled. This also holds true in the case $D>r$, with the roles of put and call and upper and lower boundaries reversed, but not for the case $D=r$ when both boundaries start from $E$. Because of this, for $D \neq r$, as $\tau \rightarrow 0$ the value of an American straddle at leading order is the sum of the values of an American call and an American put. We would stress that for larger times, the two boundaries are coupled together, and the value of an American straddle will be less than the sum of the values of an American call and an American put.

Proceeding with the analysis, we now substitute the series (4.1) into (3.1)-(3.4) and expand and collect powers of $\tau$. To evaluate the integrals on the right-hand sides of (3.1)(3.4), we make the change of variable $\zeta=\tau \eta$, which enables us to pull the $\tau$ dependence outside of the integrals when we expand. Considering first the upper boundary, from (3.1), (3.3), at leading order we find

$$
\begin{gathered}
\int_{0}^{1}\left[\sqrt{\frac{1-\eta}{2 \pi}} \exp \left(-\frac{x_{u 1}^{2}(1-\sqrt{\eta})}{2 \sigma^{2}(1+\sqrt{\eta})}\right)-\frac{x_{u 1}}{2 \sigma} \operatorname{erfc}\left(\frac{x_{u 1}}{\sigma \sqrt{2}} \sqrt{\frac{1-\sqrt{\eta}}{1+\sqrt{\eta}}}\right)\right] d \eta=0, \\
\int_{0}^{1}\left[\frac{x_{u 1}}{\sigma} \sqrt{\frac{2 \eta}{\pi(1-\eta)}} \exp \left(-\frac{x_{u 1}^{2}(1-\sqrt{\eta})}{2 \sigma^{2}(1+\sqrt{\eta})}\right)-\operatorname{erfc}\left(\frac{x_{u 1}}{\sigma \sqrt{2}} \sqrt{\frac{1-\sqrt{\eta}}{1+\sqrt{\eta}}}\right)\right] d \eta=0 .
\end{gathered}
$$

These two equations (4.2) have a numerical root $x_{u 1}=0.6388 \sigma$ which agrees with the value in [1] for the call and also with the one in [8], where this coefficient was first reported. Continuing with our expansion, at the next order, we find a numerical value of $x_{u 2}=-0.2898(r-D)$, again in very good agreement with the value reported in [1] for the call. Since the decoupled equations for the upper free boundary are identical to those for the call, it follows that all of the coefficients in the series for $x_{u}$ will be identical to those for the call.

Turning now to the lower boundary, we will attempt to use the series (4.1) for $x_{l}$ and again make the change of variable $\zeta=\tau \eta$ in (3.2), (3.4). At leading order, we find

$$
\begin{gathered}
\frac{\sigma}{\sqrt{2 \pi}} \exp \left(-\frac{x_{l 1}^{2}}{2 \sigma^{2}}\right)+\frac{x_{l 1}}{2} \operatorname{erfc}\left(-\frac{x_{l 1}}{\sqrt{2} \sigma}\right)=0, \\
\operatorname{erfc}\left(-\frac{x_{l 1}}{\sqrt{2} \sigma}\right)=0
\end{gathered}
$$


We note that each of these equations occurs a power of $\tau$ earlier than that for the upper boundary, which occurs because we cannot replace the error functions on the left-hand sides of (3.2), (3.4) as we did with (3.1), (3.3). For (4.3) to have a solution requires that $x_{l 1}=-\infty$, which suggests that the series (4.1) is inappropriate for $x_{l}(\tau)$, and we will instead suppose that

$$
x_{l}(\tau) \sim \sum_{n=0}^{\infty} x_{\ln }(\tau) \tau^{n / 2}
$$

so that the coefficients in the series are functions of $\tau$, and in turn expand the $x_{\ln }(\tau)$ themselves as series in an unknown function $f(\tau)$, which is assumed to be small,

$$
x_{l 1}(\tau) \sim \sqrt{f(\tau)} \sum_{m=0}^{\infty} x_{l 1}^{(m)}(f(\tau))^{-m} .
$$

We need to solve for $f(\tau)$ as part of the solution process. Using this new series (4.4), (4.5), we need to balance the leading-order terms from (3.2), which tells us that

$$
\begin{aligned}
& \frac{\tau^{1 / 2}(D-r)}{2} \int_{0}^{1} \operatorname{erfc}\left(-\frac{x_{l 1}(\tau)-\sqrt{\eta} x_{l 1}(\tau \eta)}{\sigma \sqrt{2(1-\eta)}}\right) d \eta \\
& \quad=-\frac{\sigma}{\sqrt{2 \pi}}\left[\frac{x_{l 1}(\tau) \sqrt{\pi}}{\sqrt{2} \sigma} \operatorname{erfc}\left(-\frac{x_{l 1}(\tau)}{\sqrt{2} \sigma}\right)+\exp \left(-\frac{x_{l 1}^{2}(\tau)}{2 \sigma^{2}}\right)\right] .
\end{aligned}
$$

Since we expect $x_{l 1}(\tau)<0$, as $\tau \rightarrow 0$, the right-hand side of (4.6) tends to

$$
-\frac{\sigma^{3}}{\sqrt{2 \pi} x_{l 1}^{(0) 2} f(\tau)} \exp \left(-\frac{x_{l 1}^{(0) 2} f(\tau)}{2 \sigma^{2}}-\frac{x_{l 1}^{(0)} x_{l 1}^{(1)}}{\sigma^{2}}\right) .
$$

To evaluate the left-hand side of (4.6), we make the change of variable $\eta=1-\xi / f(\tau)$ to enable us to strip the $\tau$ dependence out of the integral, and we note that $\int_{0}^{1} d \eta$ becomes $\int_{0}^{1 / f(\tau)} d \xi / f(\tau) \rightarrow \int_{0}^{\infty} d \xi / f(\tau)$. In the limit, the left-hand side of $(4.6)$ becomes

$$
\frac{\tau^{1 / 2}(D-r)}{2 f(\tau)} \int_{0}^{\infty} \operatorname{erfc}\left(-\frac{x_{l 1}^{(0)} \sqrt{\xi}}{\sqrt{8} \sigma}\right) d \xi \sim \frac{2 \tau^{1 / 2} \sigma^{2}(D-r)}{x_{l 1}^{(0) 2} f(\tau)},
$$

and from (4.7), (4.8), our leading-order equation is therefore

$$
\exp \left(-\frac{x_{l 1}^{(0) 2} f(\tau)}{2 \sigma^{2}}\right)=\left[\frac{2 \sqrt{2 \pi}(r-D)}{\sigma} \exp \left(\frac{x_{l 1}^{(0)} x_{l 1}^{(1)}}{\sigma^{2}}\right)\right] \sqrt{\tau}
$$

This has a solution $x_{l 1}^{(0)}=-\sigma, f(\tau)=-\ln \tau$, and $x_{l 1}^{(1)}=-\sigma \ln [2 \sqrt{2 \pi}(r-D) / \sigma]$. This $\log$ arithmic behavior is exactly what we would expect, since it is well known $[3,18]$ that this is the behavior for the put with $D<r$, and indeed both $x_{l 1}^{(0)}$ and $x_{l 1}^{(1)}$ are the same as the 
values for a put, so at leading order, the lower boundary behaves like that of the put. In a sense, this is a little surprising: if we decompose the European straddle component of (2.8) into a European put and call, for the case $D<r$, we would expect the put to be too far out of the money to contribute on the upper boundary close to expiry, which is why the upper boundary is identical to that of a call for $r<D$, but since the lower boundary starts at the strike price $E$, there should be a contribution from the call on the lower boundary, but our analysis indicates that such a contribution is not at leading order; however, we believe it will enter at a later power of $\tau$ since the decoupled equations for the lower boundary differ slightly from those for the put.

Similarly, balancing the leading-order terms from (3.4) yields the same equation (4.9) as above. Hence for $D<r$, close to expiry, the free boundary is of the form

$$
\begin{gathered}
x_{u}(\tau) \sim x_{u 1} \tau^{1 / 2}+x_{u 2} \tau+\cdots \\
x_{l}(\tau) \sim \tau^{1 / 2} \sqrt{-\ln \tau}\left[x_{l 1}^{(0)}+x_{l 1}^{(1)}(-\ln \tau)^{-1}+\cdots\right]+\cdots .
\end{gathered}
$$

As might be expected, close to expiry, the upper boundary behaves exactly like the call boundary, while the lower boundary behaves at leading order like the put boundary.

Case $4.2(D>r)$. The free boundary starts at $S_{l 0}=r E / D$ and $S_{u 0}=E$, which is the opposite of the case $D<r$. As with that case, if we take the limit $\tau \rightarrow 0$, the four equations (3.1)-(3.4) decouple into a pair of equations involving only $x_{u}$ but not $x_{l}$, and a pair of equations involving only $x_{l}$ but not $x_{u}$. From our analysis for the case $D<r$ and from symmetry, in the limit $\tau \rightarrow 0$ we expect the lower boundary to behave exactly like the boundary for the put, and the upper boundary to behave at leading order like the boundary for the call, and this is exactly what happens. The actual analysis for this case is essentially the same as for $D<r$, but with the roles of put and call and upper and lower boundaries reversed, and so the details are omitted.

For $D<r$, close to expiry, the free boundary is of the form

$$
\begin{gathered}
x_{l}(\tau) \sim x_{l 1} \tau^{1 / 2}+x_{l 2} \tau+\cdots \\
x_{u}(\tau) \sim \tau^{1 / 2} \sqrt{-\ln \tau}\left[x_{u 1}^{(0)}+x_{u 1}^{(1)}(-\ln \tau)^{-1}+\cdots\right]+\cdots
\end{gathered}
$$

For the lower boundary, the numerical root $x_{l 1}=0.6388 \sigma$ for $D>r$ is minus the value of $x_{u 1}$ found for $D<r$, which agrees with the value for the put with $D>r$ reported in the literature, while the numerical root of $x_{12}=-0.2898(r-D)$ is the same as the value of $x_{u 2}$ found earlier for $D<r$, although of course $r-D$ will be negative when $D>r$.

For the upper boundary, $x_{u 1}^{(0)}=\sigma, f(\tau)=-\ln \tau$, and $x_{u 1}^{(1)}=-\sigma \ln [2 \sqrt{2 \pi}(D-r) / \sigma]$, so that $x_{u 1}^{(0)}$ is minus the value found for $x_{l 1}^{(0)}$ when $D<r$, and $r$ and $D$ are interchanged in $x_{u 1}^{(1)}$ compared to $x_{l 1}^{(1)}$. This matches the behavior reported for the call with $D>r$ and indicates that the contribution from the put on the upper boundary is not at leading order. As for $D<r$, the upper and lower boundaries behave like those for the call and put.

Case $4.3(D=r)$. The free boundary starts at $S_{l 0}=S_{u 0}=E$, and (3.1)-(3.4) no longer decouple in the limit $\tau \rightarrow 0$. We recall that for $D<r$, we found $x_{l 1}^{(1)}=-\sigma \ln [2 \sqrt{2 \pi}(r-D) / \sigma]$, while for $D>r$, we had $x_{u 1}^{(1)}=-\sigma \ln [2 \sqrt{2 \pi}(D-r) / \sigma]$; both of which are problematic 
when $D=r$. For this case, intuitively we would expect both the put to influence the upper boundary and the call to influence the lower boundary.

We will attempt to proceed with the analysis as before, and substitute the series (4.1) into (3.1)-(3.4). At leading order, we once again arrive at (4.3) and their counterparts on the upper boundary, and these equations have a solution $x_{l 1}=-\infty$ and $x_{u 1}=\infty$. Once again, this is not possible and again suggests that we need to use the series (4.4), (4.5) for $x_{l}$ and a similar series for $x_{u}$.

At the upper boundary, we need to balance the leading-order terms from (3.1), which tells us that

$$
\begin{aligned}
& \tau r \int_{0}^{1}\left(x_{u 1}(\tau)\left[\operatorname{erfc}\left(\frac{x_{u 1}(\tau)-\sqrt{\eta} x_{u 1}(\tau \eta)}{\sigma \sqrt{2(1-\eta)}}\right)+\operatorname{erfc}\left(\frac{x_{u 1}(\tau)-\sqrt{\eta} x_{l 1}(\tau \eta)}{\sigma \sqrt{2(1-\eta)}}\right)\right]\right. \\
& +\frac{\sigma \sqrt{1-\eta}}{\sqrt{2 \pi}}\left[\exp \left(-\frac{\left(x_{u 1}(\tau)-\sqrt{\eta} x_{u 1}(\tau \eta)\right)^{2}}{2 \sigma^{2}(1-\eta)}\right)\right. \\
& \left.+\exp \left(-\frac{\left(x_{u 1}(\tau)-\sqrt{\eta} x_{l 1}(\tau \eta)\right)^{2}}{2 \sigma^{2}(1-\eta)}\right)\right] d \eta \\
& =\frac{\sigma \sqrt{2}}{\sqrt{\pi}}\left[\frac{x_{u 1}(\tau) \sqrt{\pi}}{\sqrt{2} \sigma} \operatorname{erfc}\left(\frac{x_{u 1}(\tau)}{\sqrt{2} \sigma}\right)-\exp \left(-\frac{x_{u 1}^{2}(\tau)}{2 \sigma^{2}}\right)\right] \text {. }
\end{aligned}
$$

On the lower boundary, balancing the leading-order terms from (3.2) gives (4.12) but with $x_{u 1}$ replaced by $-x_{l 1}$ and vice versa, which again tells us that $x_{l 1}=-x_{u 1}$, as might be expected from put-call symmetry for American options [7, 15, 19]. Using this, (4.12) becomes

$$
\begin{aligned}
& \tau r \int_{0}^{1}\left(\frac{x_{u 1}(\tau)}{2}\left[\operatorname{erfc}\left(\frac{x_{u 1}(\tau)-\sqrt{\eta} x_{u 1}(\tau \eta)}{\sigma \sqrt{2(1-\eta)}}\right)+\operatorname{erfc}\left(\frac{x_{u 1}(\tau)+\sqrt{\eta} x_{u 1}(\tau \eta)}{\sigma \sqrt{2(1-\eta)}}\right)\right]\right. \\
& +\frac{\sigma \sqrt{1-\eta}}{\sqrt{2 \pi}}\left[\exp \left(-\frac{\left(x_{u 1}(\tau)-\sqrt{\eta} x_{u 1}(\tau \eta)\right)^{2}}{2 \sigma^{2}(1-\eta)}\right)\right. \\
& \left.\left.+\exp \left(-\frac{\left(x_{u 1}(\tau)+\sqrt{\eta} x_{u 1}(\tau \eta)\right)^{2}}{2 \sigma^{2}(1-\eta)}\right)\right]\right) d \eta \\
& =\frac{\sigma \sqrt{2}}{\sqrt{\pi}}\left[\frac{x_{u 1}(\tau) \sqrt{\pi}}{\sqrt{2} \sigma} \operatorname{erfc}\left(\frac{x_{u 1}(\tau)}{\sqrt{2} \sigma}\right)-\exp \left(-\frac{x_{u 1}^{2}(\tau)}{2 \sigma^{2}}\right)\right] \text {. }
\end{aligned}
$$

This differs somewhat from the equations we found for $D<r$ and $D>r$ because of the presence of functions involving $x_{u 1}(\tau)+\sqrt{\eta} x_{u 1}(\tau \eta)$ rather than $x_{u 1}(\tau)-\sqrt{\eta} x_{u 1}(\tau \eta)$, so that these equations contain both $x_{u 1}$ and $x_{l 1}$. However, if we again make the substitution $\eta=1-\xi / f(\tau)$, then we can show that as $\tau \rightarrow 0$, the terms in (4.13) involving $x_{u 1}(\tau)+\sqrt{\eta} x_{u 1}(\tau \eta)$ can be neglected compared to those involving $x_{u 1}(\tau)-\sqrt{\eta} x_{u 1}(\tau \eta)$, 
and therefore the leading-order balance is

$$
\exp \left(-\frac{x_{u 1}^{(0) 2} f(\tau)}{2 \sigma^{2}}\right) \sim\left[\frac{r x_{u 1}^{(0)} \sqrt{2 \pi}}{\sigma} \exp \left(\frac{x_{u 1}^{(0)} x_{u 1}^{(1)}}{\sigma^{2}}\right)\right] \tau \sqrt{f(\tau)}
$$

which has a solution $x_{u 1}^{(0)}=\sigma, f(\tau)=W_{L}\left(\tau^{-2}\right)$, and $x_{u 1}^{(1)}=-\sigma \ln [r \sqrt{2 \pi}]$, where $W_{L}$ is the Lambert $\mathrm{W}$ function which obeys $W_{L}(x) e^{W_{L}(x)}=x$. Since $x_{l 1}=-x_{u 1}$, it follows that $x_{l 1}^{(0)}=-\sigma$ and $x_{l 1}^{(1)}=\sigma \ln [r \sqrt{2 \pi}]$. If we compare this to the values for a vanilla call or put, we find the solution has the same form but that the call and put have an additional factor of 2 inside the logarithm that is not present in $x_{l 1}^{(1)}$ and $x_{u 1}^{(1)}$, and this demonstrates that the put-like element has influenced the upper boundary and the call-like element the lower boundary. Intuitively, one would expect an American straddle to be less likely than an American call to exercised early as a call, because the payoff from continuing to hold is sweeter for the straddle, and this seems to be true close to expiry for the case $D=r$.

If we take a similar approach with the integral equations from the delta on the boundaries, we arrive at the same balance (4.14) as above. Hence for $D=r$, close to expiry, the free boundary is of the form

$$
x_{u}(\tau) \sim-x_{l}(\tau) \sim \tau^{1 / 2} \sqrt{W_{L}\left(\tau^{-2}\right)}\left[x_{u 1}^{(0)}+x_{u 1}^{(1)}\left(W_{L}\left(\tau^{-2}\right)\right)^{-1}+\cdots\right]+\cdots
$$

The upper and lower boundaries behave qualitatively like those for the call and put, respectively, but the coefficients differ, indicating that even at leading-order close to expiry, when $r=D$, the American straddle is worth less than a put and call combined, which is what one would expect intuitively since a combination of a put and a call carries the right to exercise either or both of the options while the straddle carries the right to exercise only one.

\section{Discussion}

In the previous sections, we studied the American straddle and were able to use a technique due to [16] to derive an integral expression for the value of such a straddle similar to that found in the literature for American calls and puts $[6,11,16,20]$. An American straddle is an option which allows the holder to either buy or sell (but not both) the underlying stock at the strike price at or before the expiry of the option, and this right to early exercise leads to a free boundary problem with not one but two free boundaries, a lower one $S_{l}(\tau)$ on which an exercise as a put is optimal and an upper one $S_{l}(\tau)$ on which an exercise as a call is optimal. The free boundaries for the straddle differ from those for the call and the put, so that an American straddle differs from the combination of an American call and put.

We were also able to derive a set of four coupled (Volterra of the second kind) integral equations governing the location of the free boundary, and solve these equations asymptotically to find expressions for the location of the free boundary. We found that there were three different cases depending on the relative values of the risk-free rate $r$ and the dividend yield $D$. Writing $S_{u}=S_{u 0} e^{x_{u}}$ and $S_{l}=S_{l 0} e^{x_{l}}$, we found that when $D<r, x_{u}$ 
behaved like $\tau^{1 / 2}$ as $\tau \rightarrow 0$ while $x_{l}$ behaved like $\sqrt{-\tau \ln \tau}$ (4.10), with the situation reversed when $D>r$ (4.11), and when $D=r$ and both free boundaries start from the strike price, $x_{u}$ and $x_{l}$ both behaved like $\sqrt{\tau W_{L}\left(\tau^{-2}\right)}(4.15)$.

It behooves us to say a few words about this behavior close to expiry. As we mentioned in the previous section, in this limit, we would expect the behavior of an upper boundary starting above the strike (the case $D<r$ ) to behave like the call as it will not feel the influence of the out of the money put close to expiry, and similarly we would expect the behavior of a lower boundary starting below the strike (the case $D>r$ ) to behave like the put as it will not feel the influence of the out of the money call close to expiry, and both of these aspects were observed in the previous section.

However, we would expect the behavior of an upper boundary starting at the strike (the cases $D \leq r$ ) to behave qualitatively but not quantitatively like the call as it should feel the influence of the money put close to expiry, with a similar relation between the behavior of a lower boundary starting at the strike (the cases $D \geq r$ ) and the put, and these effects can indeed be seen in the case $D=r$ where the coefficients $x_{u 1}^{(1)}$ and $x_{l 1}^{(1)}$ differ from their counterparts for the call and put, although the behavior is qualitatively the same. However, for the cases $D \neq r$, the effect cannot be seen at leading order, which is slightly surprising but presumably the effect would occur at a subsequent order.

Finally, we would mention that since this problem has not one but two free boundaries, our ability to tackle such a problem demonstrates the power of the integral equation approach.

\section{References}

[1] G. Alobaidi and R. Mallier, Asymptotic analysis of American call options, International Journal of Mathematics and Mathematical Sciences 27 (2001), no. 3, 177-188.

[2] L Laplace transforms and the American straddle, Journal of Applied Mathematics 2 (2002), no. 3, 121-129.

[3] G. Barles, J. Burdeau, M. Romano, and N. Samsoen, Critical stock price near expiration, Mathematical Finance 5 (1995), no. 2, 77-95.

[4] G. Barone-Adesi and R. J. Elliot, Free boundary problems in the valuation of securities, Working Paper, University of Alberta, Alberta, 1989.

[5] F. Black and M. Scholes, The pricing of options and corporate liabilities, Journal of Political Economy 81 (1973), 637-659.

[6] P. Carr, R. Jarrow, and R. Myneni, Alternative characterizations of the American put option, Mathematical Finance 2 (1992), no. 2, 87-106.

[7] M. Chesney and R. Gibson, State space symmetry and two-factor option pricing models, Advances in Futures and Options Research 8 (1993), 85-112.

[8] J. N. Dewynne, S. D. Howison, I. Rupf, and P. Wilmott, Some mathematical results in the pricing of American options, European Journal of Applied Mathematics 4 (1993), no. 4, 381-398.

[9] R. Geske and H. Johnson, The American put option valued analytically, Journal of Finance 39 (1984), 1511-1524.

[10] J.-Z. Huang, M. G. Subrahamanyan, and G. G. Yu, Pricing and hedging American options: a recursive investigation method, The Review of Financial Studies 9 (1998), no. 1, 277-300.

[11] S. D. Jacka, Optimal stopping and the American put, Mathematical Finance 1 (1991), no. 2, 1-14.

[12] F. Jamshidian, An analysis of American options, Working Paper, Merrill Lynch Capital Markets, New York, 1989. 


\section{The American straddle close to expiry}

[13] N. Ju, Pricing by American option by approximating its early exercise boundary as a multipiece exponential function, Review of Financial Studies 11 (1998), no. 3, 627-646.

[14] V. A. Kholodnyi, A nonlinear partial differential equation for American options in the entire domain of the state variable, Nonlinear Analysis 30 (1997), no. 8, 5059-5070.

[15] V. A. Kholodnyi and J. F. Price, Foreign Exchange Option Symmetry, World Scientific, New Jersey, 1998.

[16] I. J. Kim, The analytic valuation of American options, Review of Financial Studies 3 (1990), no. 4, $547-572$.

[17] I. I. Kolodner, Free boundary problem for the heat equation with applications to problems of change of phase. I. General method of solution, Communications on Pure and Applied Mathematics 9 (1956), 1-31.

[18] R. Mallier and G. Alobaidi, The American put option close to expiry, Acta Mathematica Universitatis Comenianae. New Series 73 (2004), no. 2, 161-174.

[19] R. McDonald and M Schroder, A parity result for American options, Journal of Computational Finance 1 (1998), no. 3, 5-13.

[20] H. P. McKean Jr, Appendix: a free boundary problem for the heat equation arising from a problem in mathematical economics, Industrial Management Review 6 (1965), 32-29.

[21] R. C. Merton, Theory of rational option pricing, Journal of Economic and Management Sciences 4 (1973), 141-183.

[22] P. A. Samuelson, Rational theory of warrant pricing, Industrial Management Review 6 (1965), $13-31$.

[23] L. N. Tao, The Cauchy-Stefan problem, Acta Mechanica 45 (1982), no. 1-2, 49-64.

[24] P. van Moerbeke, On optimal stopping and free boundary problems, Archive for Rational Mechanics and Analysis 60 (1975/76), no. 2, 101-148.

Ghada Alobaidi: Department of Mathematics and Statistics, College of Arts and Sciences, American University of Sharjah, P.O. Box 26666, Sharjah, United Arab Emirates E-mail address: galobaidi@aus.edu

Roland Mallier: Department of Applied Mathematics, University of Western Ontario, London, Canada, ON N6A 5B7

E-mail address: rolandmallier@hotmail.com 